\title{
Nrf2 activation diminishes during adipocyte differentiation of ST2 cells
}

\author{
DIONYSIOS V. CHARTOUMPEKIS ${ }^{1}$, PANOS G. ZIROS ${ }^{1}$, GERASIMOS P. SYKIOTIS ${ }^{1,2}$, \\ APOSTOLOS ZARAVINOS $^{3}$, AGATHOKLIS I. PSYROGIANNIS ${ }^{1}$, VENETSANA E. KYRIAZOPOULOU ${ }^{1}$, \\ DEMETRIOS A. SPANDIDOS ${ }^{3}$ and IOANNIS G. HABEOS ${ }^{1}$ \\ ${ }^{1}$ Department of Internal Medicine, Division of Endocrinology, Medical School, University of Patras, \\ 26504 Patras; ${ }^{2}$ Department of Pharmacology, Medical School, University of Patras, 26500 Patras; \\ ${ }^{3}$ Laboratory of Virology, Medical School, University of Crete, 71110 Heraklion, Greece
}

Received July 4, 2011; Accepted July 28, 2011

DOI: $10.3892 / \mathrm{ijmm} .2011 .761$

\begin{abstract}
Adipocyte differentiation (adipogenesis) is a highly controlled process known to be affected, among other factors, by the redox status of the cell. Nrf2 (NFE2-related factor 2) is a transcription factor that orchestrates the expression of a battery of antioxidant and detoxification genes under both basal and stress conditions. The present study investigated the activation of Nrf2 during adipocyte differentiation using as a model the mouse bone marrow-derived ST2 cell line. Treatment of ST2 cells with a differentiation cocktail containing IBMX, indomethacin, hydrocortisone and insulin induced differentiation into adipocytes over 5 days. During adipogenesis, the intracellular glutathione redox potential, which is an indicator of oxidative stress levels, became steadily more oxidized, as shown by real-time measurement in differentiating ST2 cells stably transfected with a redox-sensitive Grx1-roGFP2 fusion protein. The nuclear abundance of Nrf2 was assessed by Western immunoblotting and its DNA binding activity by EMSA (electrophoretic mobility shift assay) performed on nuclear protein extracts prepared every $24 \mathrm{~h}$. The nuclear abundance of $\mathrm{Nrf} 2$ continuously decreased during adipogenesis in ST2 cells. Its DNA binding activity reached a nadir during the first two days of differentiation, after which it increased slightly without approaching its initial level. The pattern of Nrf2 DNA binding corresponded to its transcriptional activity as assessed in ST2 cells stably transfected with a reporter construct bearing a Nrf2 bind site upstream of the luciferase gene. In conclusion, the activation of Nrf2 decreased significantly during adipogenesis. The observed changes might lead to increased oxidative stress levels that could facilitate the differentiation process. These findings could shed new light on the pathogenesis of
\end{abstract}

Correspondence to: Dr Ioannis G. Habeos, Department of Internal Medicine, Division of Endocrinology, Medical School, University of Patras, 26504 Patras, Greece

E-mail: ihabeos@med.upatras.gr

Key words: Keap1, adipogenesis, oxidative stress, reactive oxygen species, glutathione obesity, antioxidant obesity, in which the adipose tissue and oxidative stress play prominent roles.

\section{Introduction}

The transcription factor Nrf2 (NFE2-related factor 2) is a member of the cap 'n' collar family which has been described as a central orchestrator of the expression of antioxidant and detoxifying genes (1-4). Under basal conditions Nrf2 is localized mainly in the cytoplasm where it binds to the Kelch-like ECH-associating protein 1 (Keap1) and is degraded by the proteasome system. Upon exposure to oxidative or electrophilic stress, Nrf2 escapes Keap1-mediated degradation and accumulates in the nucleus where it binds to antioxidant response element (ARE) sequences in the regulatory regions of its target genes to induce their expression (5).

Adipocyte differentiation (adipogenesis) is the development of fat cells from preadipocytes. It is a process that has been studied extensively and is known to be highly regulated; it is controlled by the timely and coordinated expression of transcription factors such as the CAAT/enhancer binding protein (C\EBP) and the peroxisome proliferator-activated receptor (PPAR) families (6-8). The role of the redox status of the cell has recently emerged as a major factor during adipogenesis (9). Oxidative stress is defined as an imbalance of pro-oxidants and antioxidants and is mainly associated with increased production of reactive oxygen species (ROS) (10). ROS levels increase during adipogenesis in models such as 3T3-L1 cell line $(11,12)$, and indeed seem to facilitate adipocyte differentiation (9). Consistently, the intracellular redox-sensitive couples such as the glutathione (GSH/GSSG) and cysteine (Cys/CySS) systems shift towards more oxidized states during adipogenesis (13).

Given the role of ROS in adipogenesis and the antioxidant actions of Nrf2 as a master regulator of antioxidant gene expression, a plausible hypothesis would be that Nrf2 is implicated in the redox-associated adipogenesis process. Some studies have described the effect of the Nrf2 deletion on adipocyte differentiation with inconsistent results $(14,15)$, but they have not investigated whether Nrf2 undergoes changes with regard to its nuclear abundance, DNA binding, and transcriptional activity during adipogenesis. Obesity, a deadly epidemic in 
modern societies (16) is characterized by increased lipogenesis and adipogenesis (17). Thus, elucidating further the mechanisms of adipocyte differentiation and describing novel factors implicated in its regulation is of major importance. The present study assessed the activation status of Nrf 2 during adipocyte differentiation using the ST2 cell line as a model.

\section{Materials and methods}

Chemicals. Unless otherwise indicated, all chemicals were purchased from Sigma (St. Louis, MO).

Culture and adipocyte differentiation of ST2 cells. ST2 cells are a cloned stromal-cell line from mouse bone marrow; obtained from DSMZ (German Collection of Microorganisms and Cell Cultures). These cells have been previously shown to express functional Nrf2 $(18,19)$. ST2 cells were grown in RPMI-1640 (Biochrom, Berlin, Germany) supplemented with $10 \%$ fetal bovine serum (FBS) (Gibco, Carlsbad, CA) and antibiotics (streptomycin, penicillin) (Gibco) in a humidified $5 \% \mathrm{CO}_{2}$ atmosphere. ST2 cells were induced to differentiate to adipocytes following a previously described protocol (20) with minor modifications. Briefly, cells were plated at a density of $1 \times 10^{4} / \mathrm{cm}^{2}$ and $24 \mathrm{~h}$ later were induced to differentiate (day 0 ) into adipocytes by incubation for 2 days with a medium containing $0.5 \mathrm{mM}$ IBMX (3-isobutyl-1-methylxanthine), $60 \mu \mathrm{M}$ indomethacin, $0.5 \mu \mathrm{M}$ hydrocortisone and $5 \mathrm{nM}$ insulin in RPMI-1640 supplemented with $10 \%$ FBS. Then, the medium was changed to RPMI-1640 10\% FBS with $5 \mathrm{nM}$ insulin for another 2 days, after which it was changed to RPMI-1640 10\% FBS for one more day. After these 5 days, more than $75 \%$ of ST-2 cells had adipocyte morphology, including enlarged round cells containing lipid droplets.

Generation of stable ST-2 cell lines. The generation of ARE-ST2 cells has been previously described (18). Briefly, ST2 cells were stably transfected with a pGL2-basic-Luc plasmid bearing a single antioxidant response element (ARE) sequence upstream of the luciferase gene.

ST2 cells that stably express the Grx1-roGFP2 fusion protein were generated by viral transduction with a retroviral expression system by modifying a previously described method (21). Briefly, 293T cells (ECACC, Salisbury, UK) were seeded in $100 \mathrm{~mm}$ plates in DMEM (Gibco) 10\% FCS and were transfected with $15 \mu \mathrm{g}$ pLPCX-Grx1-roGFP2 (22), $10 \mu \mathrm{g}$ pCMV-gag-pol, and $5 \mu \mathrm{g}$ pMDG using Lipofectamine 2000 (Invitrogen) following the manufacturer's protocol. After $48 \mathrm{~h}$, the viral supernatant was filtered $(0.45 \mu \mathrm{m})$ and was concentrated by centrifugation at $25,000 \mathrm{~g}$ for $2.5 \mathrm{~h}$ at $4^{\circ} \mathrm{C}$. The viral pellet was resuspended in fresh medium and was added to ST2 cells plated in $35 \mathrm{~mm}$ dishes, and polybrene was added at a final concentration of $5 \mu \mathrm{g} / \mathrm{ml}$. After $48 \mathrm{~h}$, cells were checked under UV microscope (Eclipse TE2000-U, Nikon, Japan) for GFP expression, and puromycin $(0.4 \mu \mathrm{g} / \mathrm{ml})$ was added for selection of cells stably expressing Grx1-roGFP2.

Measurement of cellular glutathione redox potential. ST2 cells stably expressing the Grx1-roGFP2 fusion protein were plated in 96-well plates. Measurements of the emission of roGFP2 at $535 \mathrm{~nm}$ after excitation at $390 \mathrm{~nm}$ and $480 \mathrm{~nm}$ were performed in a Victor3 multilabel plate reader (Perkin Elmer, Waltham, MA). The ratio of the emission after excitation at each wavelenght $(390 / 480 \mathrm{~nm})$ was calculated. This ratio is indicative of the glutathione redox potential GSSG/GSH in the cells, with a higher ratio indicating a more oxidized state of the glutathione redox potential (22). This is a non-invasive real-time method for measuring the redox state of the redox couple GSSG/GSH, as no cell lysis or analytical separation procedures are required, and is also very sensitive to changes in the intracellular redox status.

Western immunoblotting. Nuclear cell extracts were prepared as previously described (23). Proteins were resolved on SDS $10 \%$ polyacrylamide gel followed by electrotransfer on nitrocellulose membrane (Bio-Rad, Hercules, CA). Membranes were blocked overnight with 5\% lipid-free bovine serum albumin (BSA) in Trisbuffered saline with $10 \mathrm{mM}$ Tris- $\mathrm{HCl}, \mathrm{pH} 7.4,150 \mathrm{mM} \mathrm{NaCl}$, and $0.1 \%$ Tween-20, and probed with the primary antibody at room temperature (Nrf2 C20, SP-1 PEP2, Santa Cruz Biotechnology, Santa Cruz, CA). After incubation with a horseradish peroxidaseconjugated secondary antibody (rabbit \#7074S, Cell Signaling Technology, Beverly, MA) immunoreactive bands were visualized by Amersham ECL plus (GE HealthCare, Piscataway, NJ). SP-1 (specificity protein 1) was used as a loading control as it is a transcription factor involved in early organism development (24) and its expression does not change during adipocyte differentiation. Its suitability as a loading control was also verified by staining the nitrocellulose membrane with Ponceau, another indicator of protein loading (data not shown). Densitometric analysis of Western blot assays was performed using the Image Pro Plus software (MediaCybernetics, Bethesda, MD).

Electrophoretic mobility shift assay (EMSA). EMSAs were performed as previously described (25) using the following oligonucleotides: ARE-wt, 5'-TGGGGAACCTGTGCTGA GTCACTGGAG-3' (as radiolabeled probe or cold competitor) and ARE-mut, 5'-TGGGGAACCTGTGCTAGGTCACTG GAG-3' (as cold competitor).

Luciferase assays. Luciferase assays were performed using the Steady-Glo Luciferase assay system (Promega, Madison, WI) following the manufacturer's protocol. Protein concentration measurements were performed using the Coomassie (Bradford) protein assay kit (Thermo Scientific Pierce, Watham, MA).

Statistical analyses. Each assay was performed at least three different times on different days. Data were expressed as mean \pm SD. One-way ANOVA followed by Tukey's test was performed using GraphPad Prism 5 (GraphPad Software, La Jolla, CA); $\mathrm{p}<0.05$ was considered significant. Detailed information for each experiment is given in the respective figure legend.

\section{Results}

The intracellular glutathione redox potential becomes more oxidized during adipocyte differentiation of ST2 cells. ST2 cells stably expressing the Grx1-roGFP2 protein which allows dynamic live measurements of the glutathione redox potential were used. Measurements of the glutathione redox potential were performed during adipocyte differentiation. As shown in Fig. 1, the glutathione redox potential gradually increased 


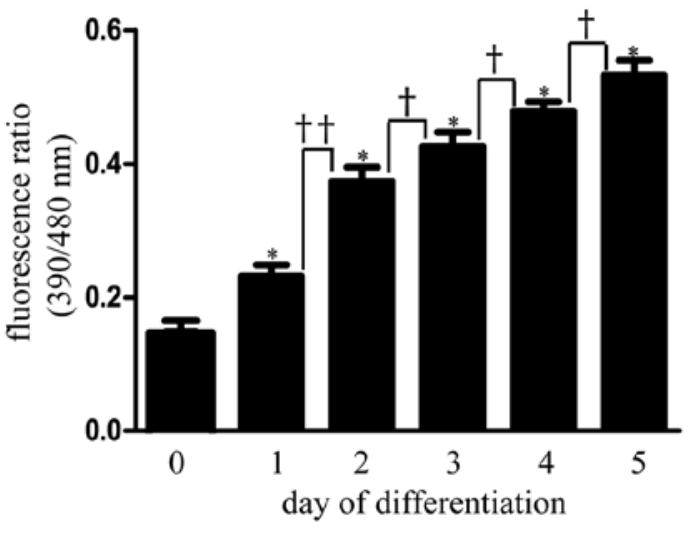

Figure 1. The intracellular glutathione redox potential becomes more oxidized during adipocyte differentiation of ST2 cells. ST2 cells stably expressing Grx1roGFP2 were induced to differentiate into adipocytes. Every $24 \mathrm{~h}$ the cells were excited with 390 and $480 \mathrm{~nm}$ lasers and the ratio of emissions in the green channel $(535 \mathrm{~nm})$ was calculated. The graph represents the mean \pm SD. Three independent experiments were performed, and in each experiment 24 technical replicates were used for each timepoint. " $\mathrm{p}<0.0001$ compared to day 0 ; ${ }^{\dagger} \mathrm{p}<0.001$; ${ }^{\dagger} \mathrm{p}<0.0001$.
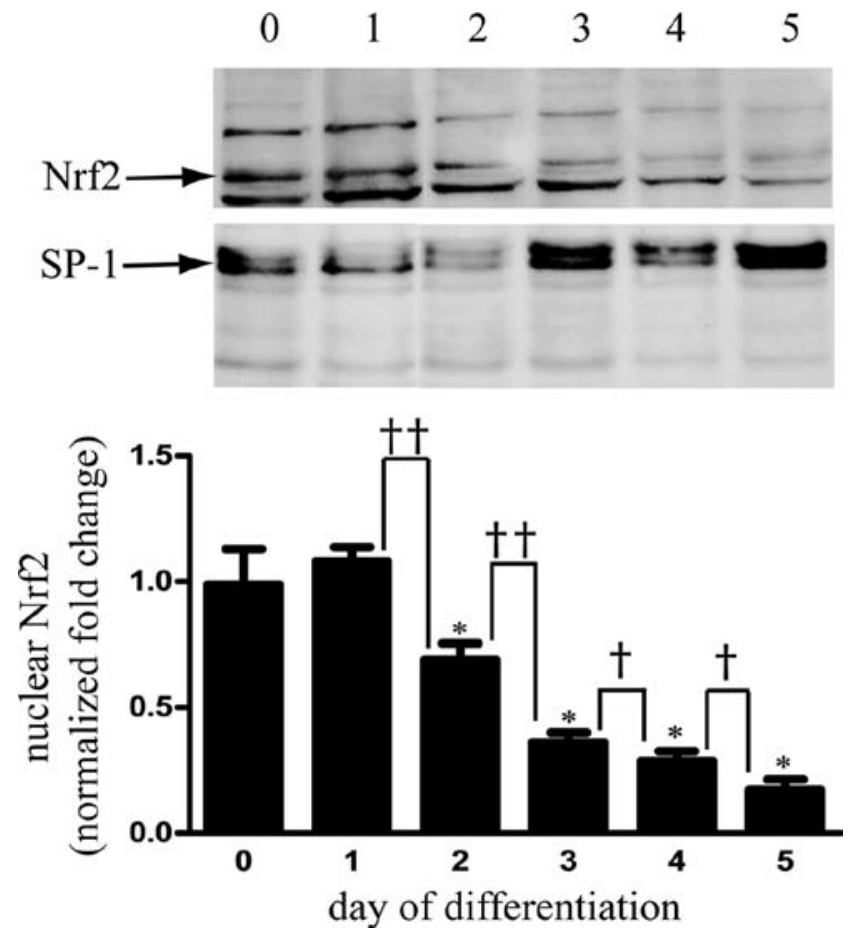

Figure 2. Nuclear Nrf2 protein abundance decreases in ST2 cells during adipocyte differentiation. ST2 cells were induced to differentiate into adipocytes. Nuclear protein extracts were prepared immediately before the initiation of differentiation (day 0 ) and every $24 \mathrm{~h}$ thereafter until day 5 . Nrf2 expression was assayed by Western immunoblotting. The nuclear abundance of the transcription factor SP-1 was employed as a loading control. The image is representative of 3 independent experiments. The graph represents the mean $\pm \mathrm{SD}$. " $\mathrm{p}<0.0001$ compared to day $0 .{ }^{\dagger} \mathrm{p}<0.05 ;{ }^{\dagger} \mathrm{p}<0.0001$.

during adipocyte differentiation of ST2 cells indicating that the cell redox status became more oxidized. As the glutathione redox potential is an index of the redox status of the cell, these findings indicate that there is a shift of the redox environment towards a more oxidized state during adipocyte differentiation.
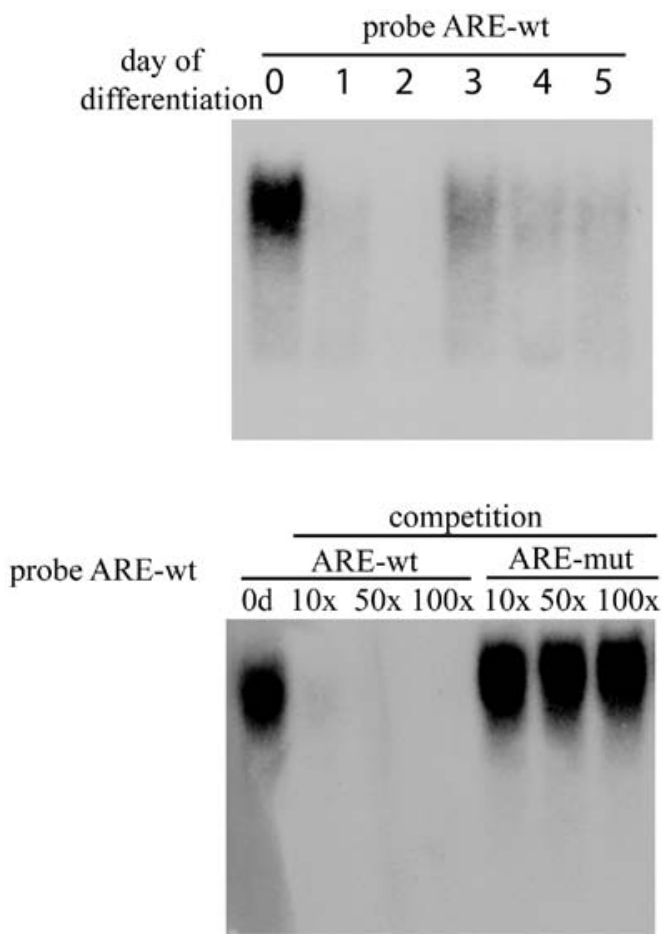

Figure 3. Nrf2 binding activity decreases during adipocyte differentiation of ST2 cells. ST2 cells were induced to differentiate into adipocytes. Nuclear protein extracts were prepared before the initiation of differentiation (day 0) and every $24 \mathrm{~h}$ thereafter until day 5, and were used for EMSAs with an AREcontaining oligonucleotide as a radiolabelled probe. The image is representative of 3 independent experiments. Binding competition experiments performed with ST2 nuclear extracts at day $0(0 \mathrm{~d})$ of differentiation (maximal Nrf2 DNA binding activity) in the presence of a 10-, 50-, or 100-fold molar excess of unlabeled ARE-wt or ARE-mut oligonucleotides. The image is representative of 3 independent experiments.

This finding is in accordance with previous studies in other models of adipocyte differentiation $(9,11,13)$, and thus validates the ST2 cell line as a model of adipogenesis in which to study redox-related phenomena and to investigate factors that may underlie their regulation.

Nuclear Nrf2 protein abundance decreases during adipocyte differentiation. ST2 cells were induced to differentiate into adipocytes and nuclear Nrf2 protein abundance was assessed by Western immunoblotting. Immediately before initiation of differentiation (day 0 ) nuclear Nrf2 protein abundance was at maximum level. Throughout the differentiation process there was a steady decrease in nuclear Nrf2 protein abundance. ST2 cells had fully differentiated into adipocytes on day 5, at which time nuclear Nrf2 levels reached a minimum (Fig. 2).

Nrf2 DNA binding activity decreases during adipocyte differentiation. Having documented decreasing nuclear Nrf2 protein abundance during adipocyte differentiation, it was investigated whether this was accompanied by changes in the binding activity of Nrf2 to the ARE. As demonstrated in Fig. 3, the ARE-binding capacity of Nrf2 decreased significantly in the first two days of differentiation, reaching a nadir on the second day; it then increased slightly without approaching its initial 


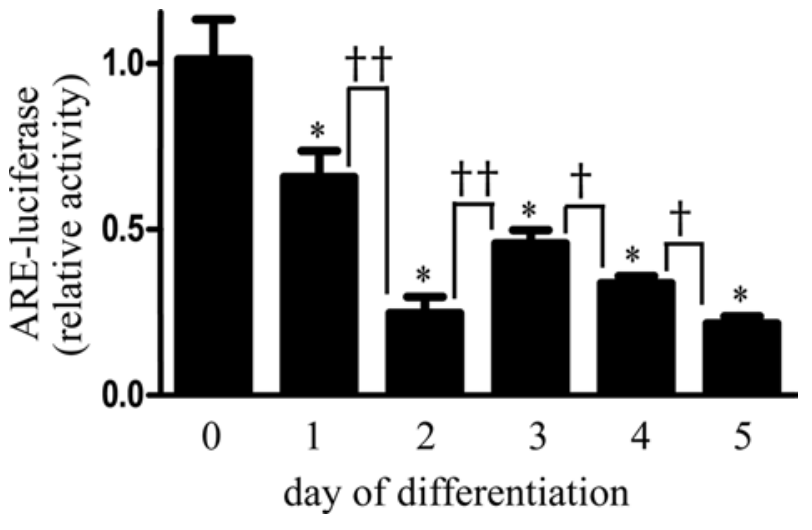

Figure 4. ARE-mediated transcription is reduced during differentiation of ARE-ST2 cells into adipocytes. ARE-ST2 cells were induced to differentiate into adipocytes and luciferase measurements were performed every $24 \mathrm{~h}$ unti day 5 of differentiation. Luciferase activity was normalized to total protein concentration. The graph represents the means \pm SD. Three independent experiments were performed, and in each experiment 8 technical replicates were used for each timepoint. ${ }^{*} \mathrm{p}<0.0001$ compared to day $0 ;{ }^{\dagger} \mathrm{p}<0.05 ;{ }^{\dagger \dagger} \mathrm{p}<0.0001$.

level. Binding competition experiments with cold (unlabeled) ARE-wt probe (Fig. 3) and with mutated (Nrf2 binding-deficient) cold probe (ARE-mut) confirmed the specificity of complex formation (as has also been shown previously in a different experimental setting) (18).

Nrf2 transcriptional activity decreases during adipocyte differentiation. To assess if the changes observed in Nrf2 binding activity in the EMSA experiments were accompanied by changes in its transcriptional activity, the ARE-ST2 cells were used. These cells stably express a plasmid bearing the ARE sequence upstream of the luciferase gene. Thus, changes in Nrf2 transcriptional activity are manifested as changes in luciferase activity. In ARE-ST2 cells induced to differentiate into adipocytes, relative luciferase activity was measured every $24 \mathrm{~h}$ during the differentiation process. As shown in Fig. 4, ARE-driven luciferase activity decreased during differentiation, reaching a minimum on day 2. A slight increase in Nrf2 transcriptional activity on day 3 was again followed by a decrease throughout the rest of the differentiation process. Therefore, both the DNA binding activity of Nrf2 (Fig. 3) and its transcriptional activity (Fig. 4) decreased substantially during adipocyte differentiation of ST2 cells.

\section{Discussion}

An increasing volume of data supports that ROS, apart from having a detrimental effect on cells (26), may also be implicated in physiological processes as signaling molecules $(27,28)$. Adipogenesis is a process which seems to be at least in part correlated with the redox status of the cell; cellular oxidative stress increases during adipocyte differentiation and appears to actually facilitate the process $(9,12)$. This study employed ST2 cells as an in vitro model of adipogenesis and assessed the glutathione redox potential during adipocyte differentiation as well as the activation status of the transcription factor Nrf2, a central regulator of antioxidant genes.

The nuclear protein abundance of Nrf2 was assessed during adipocyte differentiation of ST2 cells and was found to decrease during the course of adipogenesis. As Nrf2 is a transcription factor, it was tested whether these changes in nuclear Nrf2 abundance were accompanied by changes in its DNA binding and transcriptional activity using the EMSA and the ARE-ST2 cell line, respectively (Figs. 3 and 4). The results obtained by both techniques were similar: firstly, Nrf2 transcriptional activity was significantly lower in fully differentiated ST2 cells (day 5) compared to undifferentiated ones (day 0). Specifically, Nrf2 activity decreased sharply on the first two days of differentiation and then it increased slightly (without approaching its initial levels) on day 3, after which it decreased again. These changes in Nrf2 DNA binding capacity and transcriptional activity did not precisely match the changes in the nuclear levels of $\mathrm{Nrf} 2$. This may be due to post-translational modifications of Nrf2 (e.g. acetylation-deacetylation) that may affect its ability to bind the ARE (29). Nevertheless, comparing the undifferentiated ST2 cells (day 0 ) to the fully differentiated cells (day 5), it is evident that the differentiated cells had markedly lower Nrf2 nuclear abundance and transcriptional activity. To the best of our knowledge, this is the first study describing the changes that Nrf2 activation undergoes during adipocyte differentiation.

$\mathrm{Nrf} 2$ is a well-characterized transcriptional regulator of glutamate cysteine ligase catalytic subunit (GCLC) $(30,31)$, which is the rate-limiting enzyme in glutathione synthesis. Thus, it drives the glutathione redox pair (GSH/GSSG) towards a more reduced state, and Nrf2 knock-out mice have lower GSH levels (32). In the present study, assessments of the glutathione redox potential during adipocyte differentiation of ST-2 cells were performed to investigate whether it follows a pattern consistent with the observed changes in Nrf2 activation. Using ST2 cells stably transfected with the fusion protein ro-GFP2 that is able to detect nanomolar changes in GSSG and millimolar changes in GSH on a scale of seconds to minutes (22), the glutathione redox potential was found to become significantly more oxidized during differentiation. Hence, undifferentiated ST2 had higher Nrf2 nuclear levels, enhanced Nrf2 transcriptional activity, and a less oxidized glutathione redox potential compared to fully differentiated ST2 cells. Thus, the observed changes in glutathione redox potential, at least before and after full differentiation of ST2 cells, are consistent with the respective observed suppression in Nrf2 activation. If the increase of ROS levels was a primary event during adipocyte differentiation, it would be reasonable to expect the Keap1/Nrf2 pathway to be activated as a defense mechanism triggered by the increased oxidative stress levels. On the contrary, Nrf2 activation was found to diminish during adipogenesis, suggesting that this decrease may precede the increment in ROS and the oxidation of GSH.

The present data are in agreement with previous studies showing that ROS levels increase during adipocyte differentiation in another cellular model of adipogenesis (3T3-L1 cells) $(11,12)$, and that the glutathione redox potential shifts towards a more oxidized state during adipogenesis in human mesenchymal stem cells (13). The role of cellular redox status changes during adipogenesis has also been highlighted by a study showing that GSH depletion in 3T3-L1 cells promoted adipogenesis by enhancing the mitotic clonal expansion (MCE) phase and by activating the transcription factor $\mathrm{C} / \mathrm{EBP} \beta$, whereas $\mathrm{GSH}$ supplementation (with GSHest, a permeable compound able to increase the intracellular GSH levels) inhibited adipogenesis 
(33). Another independent study showed that ROS facilitate adipocyte differentiation in 3T3-L1 cells again by accelerating the MCE phase and activating $\mathrm{C} / \mathrm{EBP} \beta$, whereas exposure to broadly-acting antioxidants such as $\mathrm{N}$-acetyl cysteine greatly inhibited adipogenesis (9). These studies indicate that adipogenesis is facilitated by an oxidized cell redox status, and that disturbance of this status by antioxidants can compromise the process.

Given that Nrf2 is a master regulator of antioxidant gene expression whose deletion is known to increase ROS in mouse tissues (34) and embryonic fibroblasts (19), it would be reasonable to suppose that its deletion could potentially facilitate adipogenesis. To date, studies on the role of Nrf2 in adipogenesis have yielded contradicting results. On the one hand, Shin et al (15) have shown that Nrf2 deletion enhances adipogenesis in mouse embryonic fibroblasts, and that Nrf2 activation (by Keap1 deletion or treatment with CDDO-Im) disrupts adipocyte differentiation through modulation of Aryl Hydrocarbon Receptor (AhR). On the other hand, Pi et al (14) have shown that Nrf2 deletion results in impaired adipogenesis through down-regulation of PPAR $\gamma$. Furthermore, Takahashi et al (35) have shown that treatment of 3T3-L1 cells with the Nrf2 activators carnosic acid and carnosol inhibits adipogenesis and increases GSH levels. All these studies indicate that the role(s) of Nrf2 in adipogenesis is likely not limited to its effect on oxidative stress levels; rather, $\mathrm{Nrf} 2$ as a transcription factor can also have an effect on other molecules known to be involved in adipogenesis such as PPAR $\gamma$ and AhR. Therefore, the observed discrepancies in the role of Nrf 2 in adipogenesis may be due to the differential effects that Nrf2 may have on this process in different experimental systems by simultaneously controlling antioxidant genes that decrease ROS and other players (e.g. transcription factors) that are directly involved in the execution of the adipogenic program.

The present study demonstrates that Nrf2 is repressed in the differentiated adipocyte compared to the preadipocyte. The functional role of $\mathrm{Nrf} 2$ repression during adipogenesis remains to be determined. Further experiments with fine manipulation of Keap1/Nrf2 signaling are needed to elucidate the impact that changes in Nrf2 activation and cell redox potential have on adipogenesis. Another worthwhile aim of future experiments would be to clarify the mechanism(s) through which Nrf2 is repressed during adipogenesis by testing the impact that various adipogenesis-promoting factors have on Nrf2 activity.

In conclusion, the suppression of Nrf2 activity during adipogenesis may be crucial, and it might be feasible with appropriate pharmacological manipulation of Nrf2 to control the process of adipocyte differentiation. This could potentially have therapeutic implications in obesity, a disease associated with both adipogenesis (36) and increased oxidative stress (37).

\section{Acknowledgements}

We thank Dr Tobias P. Dick (German Cancer Research CenterDKFZ) for kindly providing us with the pLPCX-Grx1-roGFP2 plasmid.

\section{References}

1. Calkins MJ, Johnson DA, Townsend JA, et al: The Nrf2/ARE pathway as a potential therapeutic target in neurodegenerative disease. Antioxid Redox Signal 11: 497-508, 2009.
2. Kobayashi $M$ and Yamamoto $M$ : Nrf2-Keap1 regulation of cellular defense mechanisms against electrophiles and reactive oxygen species. Adv Enzyme Regul 46: 113-140, 2006.

3. Sykiotis GP and Bohmann D: Stress-activated cap 'n' collar transcription factors in aging and human disease. Sci Signal 3: re3, 2010.

4. Kensler TW, Wakabayashi $\mathrm{N}$ and Biswal S: Cell survival responses to environmental stresses via the Keap1-Nrf2-ARE pathway. Annu Rev Pharmacol Toxicol 47: 89-116, 2007.

5. Osburn WO and Kensler TW: Nrf2 signaling: an adaptive response pathway for protection against environmental toxic insults. Mutat Res 659: 31-39, 2008.

6. Mandrup S and Lane MD: Regulating adipogenesis. J Biol Chem 272: 5367-5370, 1997

7. Rosen ED, Walkey CJ, Puigserver P and Spiegelman BM: Transcriptional regulation of adipogenesis. Genes Dev 14: 1293-1307, 2000.

8. Rosen ED and Spiegelman BM: Molecular regulation of adipogenesis. Annu Rev Cell Dev Biol 16: 145-171, 2000.

9. Lee H, Lee YJ, Choi H, Ko EH and Kim JW: Reactive oxygen species facilitate adipocyte differentiation by accelerating mitotic clonal expansion. J Biol Chem 284: 10601-10609, 2009.

10. Jones DP: Redefining oxidative stress. Antioxid Redox Signal 8: 1865-1879, 2006

11. LeeOH,Kwon YI,HongHD,ParkCS,LeeBYandKim YC:Production of reactive oxygen species and changes in antioxidant enzyme activities during differentiation of 3T3-L1 adipocyte. J Korean Soc Appl Biol Chem 52: 70-75, 2009.

12. Ducluzeau PH, Priou M, Weitheimer M, et al: Dynamic regulation of mitochondrial network and oxidative functions during 3T3-L1 fat cell differentiation. J Physiol Biochem: doi: 10.1007/ s13105-011-0074-6, (In press).

13. Imhoff BR and Hansen JM: Differential redox potential profiles during adipogenesis and osteogenesis. Cell Mol Biol Lett 16: $149-161,2011$.

14. Pi J, Leung L, Xue P, et al: Deficiency in the nuclear factor E2-related factor-2 transcription factor results in impaired adipogenesis and protects against diet-induced obesity. J Biol Chem 285: 9292-9300, 2010.

15. Shin S, Wakabayashi N, Misra V, et al: NRF2 modulates aryl hydrocarbon receptor signaling: influence on adipogenesis. Mol Cell Biol 27: 7188-7197, 2007.

16. Dixon JB: The effect of obesity on health outcomes. Mol Cell Endocrinol 316: 104-108, 2010.

17. Nishimura S, Manabe I, Nagasaki M, et al: Adipogenesis in obesity requires close interplay between differentiating adipocytes, stromal cells, and blood vessels. Diabetes 56: 1517-1526, 2007.

18. Habeos IG, Ziros PG, Chartoumpekis D, Psyrogiannis A, Kyriazopoulou V and Papavassiliou AG: Simvastatin activates Keap1/Nrf2 signaling in rat liver. J Mol Med 86: 1279-1285, 2008.

19. Chartoumpekis D, Ziros PG, Psyrogiannis A, Kyriazopoulou V, Papavassiliou AG and Habeos IG: Simvastatin lowers reactive oxygen species level by Nrf2 activation via PI3K/Akt pathway. Biochem Biophys Res Commun 396: 463-466, 2010.

20. Ding J, Nagai K and Woo JT: Insulin-dependent adipogenesis in stromal ST2 cells derived from murine bone marrow. Biosci Biotechnol Biochem 67: 314-321, 2003.

21. Yang J, Friedman MS, Bian H, Crofford LJ, Roessler B and McDonagh KT: Highly efficient genetic transduction of primary human synoviocytes with concentrated retroviral supernatant. Arthritis Res 4: 215-219, 2002.

22. Gutscher M, Pauleau AL, Marty L, et al: Real-time imaging of the intracellular glutathione redox potential. Nat Methods 5: 553-559, 2008

23. Ziros PG, Gil AP, Georgakopoulos T, et al: The bone-specific transcriptional regulator $\mathrm{Cbfa} 1$ is a target of mechanical signals in osteoblastic cells. J Biol Chem 277: 23934-23941, 2002.

24. Black AR, Black JD and Azizkhan-Clifford J: Sp1 and kruppellike factor family of transcription factors in cell growth regulation and cancer. J Cell Physiol 188: 143-160, 2001.

25. Habeos I, Ziros PG, Psyrogiannis A, Vagenakis AG and Papavassiliou AG: Statins and transcriptional regulation: the FXR connection. Biochem Biophys Res Commun 334: 601-605, 2005.

26. Finkel $\mathrm{T}$ and Holbrook NJ: Oxidants, oxidative stress and the biology of ageing. Nature 408: 239-247, 2000.

27. Droge W: Free radicals in the physiological control of cell function. Physiol Rev 82: 47-95, 2002.

28. D'Autreaux B and Toledano MB: ROS as signalling molecules: mechanisms that generate specificity in ROS homeostasis. Nat Rev Mol Cell Biol 8: 813-824, 2007. 
29. Kawai Y, Garduno L, Theodore M, Yang J and Arinze IJ: Acetylation-deacetylation of the transcription factor Nrf2 (nuclear factor erythroid 2-related factor 2) regulates its transcriptional activity and nucleocytoplasmic localization. J Biol Chem 286: 7629-7640, 2010.

30. Aleksunes LM and Manautou JE: Emerging role of Nrf2 in protecting against hepatic and gastrointestinal disease. Toxicol Pathol 35: 459-473, 2007.

31. Yang H, Magilnick N, Lee C, et al: Nrf1 and Nrf2 regulate rat glutamate-cysteine ligase catalytic subunit transcription indirectly via NF-kappaB and AP-1. Mol Cell Biol 25: 5933-5946, 2005.

32. Chan JY and Kwong M: Impaired expression of glutathione synthetic enzyme genes in mice with targeted deletion of the Nrf2 basic-leucine zipper protein. Biochim Biophys Acta 1517: 19-26, 2000.

33. Vigilanza P, Aquilano K, Baldelli S, Rotilio G and Ciriolo MR: Modulation of intracellular glutathione affects adipogenesis in 3T3-L1 cells. J Cell Physiol 226: 2016-2024, 2011.
34. Beyer TA, Xu W, Teupser D, et al: Impaired liver regeneration in Nrf2 knockout mice: role of ROS-mediated insulin/IGF-1 resistance. EMBO J 27: 212-223, 2008.

35. Takahashi T, Tabuchi T, Tamaki Y, Kosaka K, Takikawa Y and Satoh T: Carnosic acid and carnosol inhibit adipocyte differentiation in mouse 3T3-L1 cells through induction of phase2 enzymes and activation of glutathione metabolism. Biochem Biophys Res Commun 382: 549-554, 2009.

36. Spiegelman BM and Flier JS: Adipogenesis and obesity: rounding out the big picture. Cell 87: 377-389, 1996.

37. Furukawa S, Fujita T, Shimabukuro M, et al: Increased oxidative stress in obesity and its impact on metabolic syndrome. J Clin Invest 114: 1752-1761, 2004. 